Nervenarzt 2022 · 93:221-222

https://doi.org/10.1007/s00115-022-01277-3

Angenommen: 17. Februar 2022

(c) The Author(s), under exclusive licence to Springer Medizin Verlag $\mathrm{GmbH}$, ein Teil von Springer Nature 2022

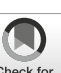

\section{Schnell wirksame Antidepressiva}

\author{
Michael Bauer ${ }^{1} \cdot$ Katharina Domschke \\ 'Klinik und Poliklinik für Psychiatrie und Psychotherapie, Universitätsklinikum Carl Gustav Carus, \\ Medizinische Fakultät, Technische Universität Dresden, Dresden, Deutschland \\ ${ }^{2}$ Klinik für Psychiatrie und Psychotherapie, Universitätsklinikum Freiburg, Medizinische Fakultät, Albert- \\ Ludwigs-Universität Freiburg, Freiburg, Deutschland
}

Mit der eher zufälligen Entdeckung der antidepressiven Eigenschaften von Imipramin im Jahre 1957 erfuhr die Therapie depressiver Störungen nicht nur erstmals eine spezifische medikamentöse Behandlungsmöglichkeit, sondern trug auch zur Formulierung der für mehrere Jahrzehnte führenden „Monoaminhypothese der Depression“ bei. Als Folge kamen zahlreiche nichtselektive Monoaminwiederaufnahmehemmer, u.a. die Gruppe der tri- und tetrazyklischen Antidepressiva, auf den Markt, die erst in den späten 1980er-Jahren durch Substanzen ergänzt wurden, die ein wesentlich selektiveres Rezeptorbindungsprofil aufwiesen, wie z.B. die Gruppe der selektiven Serotoninwiederaufnahmehemmer (SSRI) oder der Serotonin- und Noradrenalinwiederaufnahmehemmer (SNRI). Die höhere Rezeptorselektivität erbrachte den unbestrittenen großen Vorteil, dass diese neuen Substanzen eine bessere Verträglichkeit und geringe Toxizität in Überdosierung aufwiesen. Dies führte dazu, dass sie heute weltweit die Verordnungsstatistiken anführen und in Leitlinien als sog. First-line-Antidepressiva geführt werden, auch wenn sie keine höhere Wirksamkeit aufweisen als die traditionellen Antidepressivagenerationen.

Trotz dieser Fortschritte ist jedoch bis heute allen Antidepressiva gemein, dass es bis zum erwünschten Wirkeintritt gewöhnlich eine Latenz von mehreren Wochen gibt und der Anteil der Patienten mit unzureichendem Behandlungserfolg relativ hoch bleibt. Der fehlende Unterschied in diesen beiden Punkten ist der Tatsache geschuldet, dass die verfügbaren Antidepressiva wahrscheinlich über einen gemeinsamen Mechanismus, die Modulation monoami- nerger Neurotransmittersysteme (Serotonin, Noradrenalin und Dopamin), ihre Wirkung entfalten.

\section{》) Verfügbare Antidepressiva entfalten ihre Wirkung wahrscheinlich über gemeinsamen Mechanismus}

Die Entdeckung neuer pharmakologischer Wirkprinzipien antidepressiver Therapien hat vor einigen Jahren mit der Erforschung der potenziellen Rolle des glutamatergen Neurotransmittersystems in der Ätiopathogenese der Depression spürbaren Auftrieb erhalten. Unterstützt wurde diese Entwicklung wesentlich im Jahr 2000 durch eine an der renommierten Yale University in den USA durchgeführte klinische Pilotstudie, die Hinweise ergab, dass eine einmalige i.v. Infusion einer subanästhetischen Dosis von Ketamin innerhalb von $24 \mathrm{~h}$ eine mehrere Tage anhaltende antidepressive Wirkung entfaltet [1]. Das seit den 1960erJahren zugelassene Ketamin, ein bis heute gebräuchliches Anästhetikum und Analgetikum, ist ein Antagonist der glutamatergen NMDA-Rezeptoren mit der Folge einer veränderten glutamatergen Signaltransduktion als möglichem Wirkmechanismus. Die Pilotstudie aus Yale war der Ausgangspunkt für eine Vielzahl größerer und später auch placebokontrollierter Studien, die zur Entwicklung und Zulassung von Esketamin führten. In diesem Kontext entstand in der Folge auch der Begriff "Schnell wirksame Antidepressiva“" (,rapidly acting antidepressants", RAAD; [2]).

Als Folge dieser Entwicklung hat die amerikanische Arzneimittelbehörde FDA 2019 und 9 Monate später auch die Europäische Zulassungsbehörde EMA nach 
mehr als 30 Jahren erstmals wieder ein Medikament zur Behandlung schwerer, behandlungsresistenter Depressionen zugelassen. Es folgte dann eine Zulassungserweiterung für Esketamin durch die FDA (2020) und die EMA (2021) zur Akutbehandlung depressiver Notfälle, allerdings immer in Kombination mit einem Antidepressivum aus der Klasse der SSRI oder SNRI. Der Gemeinsame Bundesauschuss (G-BA) sah im Sommer 2021 entsprechend einen (in der Psychopharmakologie selten vergebenen) sog. "geringen Zusatznutzen“ für das Arzneimittel Spravato ${ }^{\circledR}$ (Wirkstoff Esketamin als Nasenspray), wenn es für die akute Kurzzeitbehandlung von Erwachsenen mit Depressionen bei einer mittelgradigen bis schweren Episode zusammen mit einem Antidepressivum eingesetzt wird. Als weitere möglicherweise schnell wirksame Antidepressiva werden Psychedelika wie der Serotoninrezeptoragonist Psilocybin, Ayahuasca oder Lysergsäurediethylamid (LSD) untersucht $[3,4]$.

Diese Ausgabe von Der Nervenarzt widmet sich in vier Beiträgen dem Thema "Schnell wirksame Antidepressiva", die in erster Linie eine aktuelle Literaturübersicht zum Inhalt haben. Der Beitrag von Gass und Kolleg*innen beschreibt die neurobiologischen Grundlagen des Glutamatsystems und die zugrunde liegenden neuropharmakologischen Effekte schnell wirksamer Antidepressiva, insbesondere von Ketamin, dem Prototyp dieser Gruppe, der bereits nach einer einmaligen i.v. Gabe zu raschen Veränderungen des glutamatergen Neurotransmittersystems führt. Die Autoren verweisen jedoch auch darauf, dass es noch andere, ebenfalls vielversprechende, potenziell schnell wirksame Antidepressiva gibt, die nicht direkt über das Glutamatsystem wirken, beispielsweise den GABARezeptormodulator Brexanolon oder den Serotoninrezeptoragonisten Psilocybin.

In der Übersicht von Vestring und Kolleg*innen wird ein Überblick über die wichtigsten Substanzen, Zielstrukturen und Entwicklungsfortschritte von NMDARezeptor-Modulatoren gegeben. Der Beitrag umfasst nicht nur die Bewertung der Studien zu (Es-)Ketamin, sondern gibt zugleich Einblick in die derzeit intensive Suche nach weiteren antidepressiv wirksamen Substanzen, die das glutamaterge
System im Hinblick auf eine antidepressive Wirkung modulieren. Die Autoren schlussfolgern, dass die Wirksamkeit der intravenösen Behandlung therapieresistenter depressiver Patienten im stationären Setting mit R-/S-Ketamin gut belegt sei und eine Off-label-Indikation darstelle.

Findeis und Kolleg*innen fassen die derzeitige Studienlage zu Nebenwirkungen und Sicherheitsaspekten zusammen, wobei die praktischen Aspekte der Ketaminbehandlung ebenfalls beleuchtet werden. Abgerundet wird die Arbeit mit der Darstellung einer 5-jährigen klinischen Erfahrung mit antidepressiver Ketaminbehandlung der Autor*innen. Anhand einer Literaturübersicht und eigener Sicherheitsdaten kommen die Autor*innen zu dem Schluss, dass nach gegenwärtigem Kenntnisstand, die Ketaminbehandlung eine sichere Methode zur Behandlung akuter Depressionen ist. Die Autor*innen weisen allerdings darauf hin, dass trotz zahlreicher kontrollierter Studien Aspekte der praktischen Anwendung im klinischen Alltag weiterhin einer kritischen Prüfung unterworfen werden müssen.

In dem Beitrag von Gründer und Kolleg*innen "Sind Psychedelika schnellwirksame Antidepressiva?" werden schließlich die bislang zu diesem Thema publizierten Studien, die sämtlich zu Psilocybin und in noch kleinen Patientenkollektiven (gesamtes $n<200$ ) durchgeführt wurden, vorgestellt. Die bisherige Studienlage legt eine überzeugende und sehr rasch - teilweise innerhalb von $24 \mathrm{~h}$ - einsetzende Wirkung bei Depression, therapieresistenter Depression und Depression im Rahmen lebensbedrohlicher Krebserkrankungen nahe. Es ist allerdings zu bemerken, dass sich Psychedelika nicht als Notfallmedikamente zum raschen Einsatz in Krisensituationen eignen, da die Behandlung mit diesen Substanzen immer in einen elaborierten psychotherapeutischen Kontext eingebettet werden muss.

Es ist erfreulich, dass in Deutschland nun nach langer Abstinenz mit Esketamin ein neues, vor allem rasch wirksames Antidepressivum für die klinische Anwendung zur Verfügung steht. Wie sich Esketamin und eventuell ähnlich wirkende Substanzen in der breiten klinischen Anwendung bewähren, muss sich allerdings in den kommenden Jahren zeigen. Fragen der
Langzeitbehandlung im Hinblick auf Wirkung und Nebenwirkungen sind ebenso zu beantworten. Weitere Substanzen mit Wirkung am NMDA-Rezeptoren oder auch Psychedelika wie Psilocybin befinden sich erst in der frühen klinischen Entwicklung und stehen deshalb nicht unmittelbar vor einer klinischen Zulassung.

Korrespondenzadresse

Prof. Dr. Dr. Michael Bauer

Klinik und Poliklinik für Psychiatrie und

Psychotherapie, Universitätsklinikum

Carl Gustav Carus, Medizinische Fakultät,

Technische Universität Dresden

Dresden, Deutschland

michael.bauer@ukdd.de

Interessenkonflikt. M. Bauer: Beratertätigkeit: Biogen, GHResearch, Livanova GmbH, Novartis, Sunovion. M. Bauer und K. Domschkesind Mitglieder des Steering Committee Neurosciences, Janssen-Cilag GmbH.

\section{Literatur}

1. Berman RM, Cappiello A, Anand A, Oren DA, Heninger GR, Charney DS, Krystal JH (2000) Antidepressant effects of ketamine in depressed patients. Biol Psychiatry 47:351-354

2. Wohleb ES, Gerhard D, Thomas A, Duman RS (2017) Molecular and cellular mechanisms of rapid-acting antidepressants Ketamine and scopolamine. Curr Neuropharmacol 15:11-20

3. Goldberg SB, Shechet B, Nicholas CR, Ng CW, Deole G, Chen Z, Raison CL (2020) Post-acute psychological effects of classical serotonergic psychedelics: a systematic review and metaanalysis. Psychol Med 50:2655-2666

4. Romeo B, Karila L, Martelli C, Benyamina A (2020) Efficacy of psychedelic treatments on depressive symptoms: a meta-analysis. J Psychopharmacol 34:1079-1085 